\title{
Evaluation of the novel near-infrared fluorescence tracers pullulan polymer nanogel and indocyanine green $/ \gamma$-glutamic acid complex for sentinel lymph node navigation surgery in large animal models
}

\author{
Seong-Ho Kong $\cdot$ Young-Woock Noh $\cdot$ Yun-Suhk Suh • \\ Hye Sun Park • Hyuk-Joon Lee • Keon Wook Kang • \\ Hee Chan Kim • Yong Taik Lim • Han-Kwang Yang
}

Received: 14 August 2013/ Accepted: 13 January 2014/Published online: 31 January 2014

(c) The International Gastric Cancer Association and The Japanese Gastric Cancer Association 2014

\begin{abstract}
Background This study aimed to examine tracers designed to overcome the disadvantages of indocyanine green (ICG), which disperses quickly to multiple lymph nodes, using a near-infrared (NIR) imaging system in animal models.

Methods Diluted ICG, ICG/poly- $\gamma$-glutamic acid (PGA) complex, and IRDye900-conjugated pullulan-cholesterol nanoprobe "near-infrared polynagogel" (NIR-PNG) were injected into the stomachs of dogs and pigs, and the patterns of dispersion were observed using an NIR imaging system. To compare retention times, fluorescence signals were evaluated in the stomach and small bowel of animals 1 week after injection.
\end{abstract}

S.-H. Kong and Y.-W. Noh contributed equally to this work.

Electronic supplementary material The online version of this article (doi:10.1007/s10120-014-0345-3) contains supplementary material, which is available to authorized users.

S.-H. Kong · Y.-S. Suh · H.-J. Lee · H.-K. Yang

Department of Surgery, Seoul National University Hospital,

Seoul, Korea

\section{Y.-W. Noh}

Infection and Immunity Research Center, Korea Research

Institute of Bioscience and Biotechnology, Daejeon, Korea

H. S. Park

Division of Magnetic Resonance Research,

Korea Basic Science Institute, Cheongwon, Korea

H.-J. Lee · K. W. Kang · H.-K. Yang ( $ه)$

Cancer Research Institute, Seoul National University Hospital,

101 Daehang-Ro, Jongno-Gu, Seoul 110-744, Korea

e-mail: hkyang@snu.ac.kr
Results A diluted concentration $(\sim 0.1 \mathrm{mg} / \mathrm{ml})$ of ICG was optimal for NIR imaging compared with the conventional concentration $(5 \mathrm{mg} / \mathrm{ml})$ for visual inspection. When injected into the stomach, the signals of ICG and ICG/PGA complex were relatively large at the injection site, and signals were detected at multiple sentinel nodes and lymph nodes beyond them. The NIR-PNG signal intensity was relatively small at the injection site and limited to only one sentinel node with no additional node. When evaluated 1 week after injection, only the NIR-PNG signal was detected in the canine stomach, and the signal intensity at the lymph nodes of the porcine small bowel was the highest with NIR-PNG, followed by ICG/PGA complex and finally ICG.

Conclusion NIR-PNG showed the best characteristics of less dispersion and longer retention in the sentinel nodes, and ICG/PGA complex remained longer than diluted ICG. These tracers could potentially be used as optimal tracers for sentinel node navigation surgery in gastric cancer.

\section{K. W. Kang}

Department of Nuclear Medicine,

Seoul National University Hospital, Seoul, Korea

H. C. Kim

Department of Biomedical Engineering,

Seoul National University Hospital, Seoul, Korea

Y. T. Lim $(\bowtie)$

SKKU Advanced Institute of Nanotechnology (SAINT),

School of Chemical Engineering, Sungkyunkwan University,

Suwon 440-746, Korea

e-mail: yongtaik@skku.edu 
Keywords Sentinel lymph node biopsy - Near-infrared . Stomach neoplasm

\section{Introduction}

Sentinel node navigation surgery (SNNS) has been proven to be effective in breast cancer and malignant melanoma $[1,2]$. Additionally, SNNS has been also applied to gastric cancer with efforts to overcome the problems of complex lymphatic structure around the stomach and significant false negativity [3]. The tracer and detection system is one of the important factors related to the accuracy of SNNS. The most commonly used tracers for sentinel node mapping are vital dyes, which are detected by visual inspection, and radioisotopes conjugated with colloid particles, which are detected by gamma probes yielding audible signals [47]. In gastric cancer, low sensitivity and rapid dispersion of vital dyes, as well as the need for strict radiohazard-proof protocols and the invisibility of radioisotopes, have been recognized as limitations [8].

The near-infrared (NIR) imaging system, using 700- to 1,000-nm wavelengths, has been introduced for sentinel node mapping as an alternative to provide better sensitivity than vital dyes without the concern for radio-hazards [9, 10]. As NIR-producing fluorophores, inorganic fluorescent semiconductor nanocrystals, also called "quantum dots," have been proposed to have many good characteristics, including very high quantum yields, reliable capability of tuning the wavelength, and remarkable resistance to photobleaching. However, quantum dots could not be readily applied to humans because they use potentially toxic materials such as cadmium, tellurium, selenium, and lead for a core, and this is a finding that is worrisome in spite of the fact that the dosage used for imaging is usually far less than those that are known to be toxic [9-12]. By contrast, organic fluorophores including indocyanine green (ICG) and modified heptamethine indocyanines have the disadvantages of lower quantum yields and high susceptibility to photobleaching but have better safety profiles. In particular, ICG was approved for use in indicator-dilution studies by the FDA in 1958, and it is known to be one of the least toxic agents ever administered to humans, with the only known adverse reaction being rare anaphylaxis [13]. ICG emits an 800- to 840-nm fluorescence signal, has been applied in several malignancies, such as breast [14-16], colon [17, 18], and vulva cancers [19], and has been reported to be more sensitive than infrared imaging in gastric cancer [20-22].

However, when ICG is used at the same concentration for visualization in gastric cancer, the problems of rapid and extensive dispersion persist in NIR imaging systems [23]. To overcome these disadvantages of ICG, we applied three strategies in the present study. First, the concentration of ICG was adjusted. Second, ICG was physically mixed with adjunctive chemicals to enhance the retention time of the tracers in the sentinel nodes. Poly- $\gamma$-glutamic acid ( $\gamma$-PGA) is produced by Bacillus subtilis during the fermentation of soybeans and is used in a broad range of industries, including the biomedical, food, and cosmetics industries, because of its minimal toxicity and immunogenicity [24, 25]. We reported that the physical mixture of ICG and $\gamma$ PGA shows higher signal intensity and a longer retention time in sentinel nodes in small animal models [26]. The third strategy was to compose a nanoparticle of optimal size (10-100 nm) [27, 28]. Pullulan, a neutral linear polysaccharide produced from starch by Aureobasidium pullulans, was used in the current study because of its water-soluble, biodegradable, nontoxic, and nonimmunogenic properties, as well as its amenability toward chemical modification [29]. We also previously reported that this pullulan-cholesterol polymer nanogel demonstrated enhanced photostability and retention time in small animals [30].

This present study aimed to examine these tracers, which used potentially safe materials in comparison with quantum dots, in large animal models to determine whether the tracers could overcome the disadvantage of ICG, which disperses quickly to multiple lymph nodes, and whether they could be clinically applicable for gastric cancer using NIR imaging systems.

\section{Materials and methods}

\section{Materials}

\section{ICG and ICG/ $\gamma-P G A$ complex}

Indocyanine green (Dongindang Pharmaceutical, Siheung, Korea) and poly- $\gamma$-glutamic acid (BioLeaders, Daejeon, Korea) were used in this study. The ICG/ $\gamma$-PGA complex was prepared in an aqueous solution by mixing ICG and $\gamma$ PGA $(0.1 \% \mathrm{w} / \mathrm{v})$ in $1 \mathrm{ml}$ water under rapid vortexing at room temperature, as previously reported [26].

\section{Pullulan-cholesterol nanogels conjugated with IRDye800 (NIR-PNG)}

Near-infrared polynagogel (NIR-PNG) was prepared in the same manner as previously reported [30]. Pullulan (Wako Pure Chemicals, Tokyo, Japan) was conjugated by aminecholesterol to produce pullulan-cholesterol conjugate, and NHS terminated IRDye800 (Li-COR, Lincoln, NE, USA) was conjugated to additional amine moieties. The size of the nanoprobe was measured by dynamic light scattering 
and transmission electron microscopy (TEM), which revealed a mean diameter of $28.3 \pm 0.50 \mathrm{~nm}$.

\section{The NIR fluorescence imaging system}

The NIR imaging system consisted of an excitation light source (780 nm, diode laser) and a cold charge-coupled device camera (Orca ERG; Hamamatsu Photonics, Hamamatsu City, Japan) with an 845-nm bandpass emission filter as previously described (Supplementary Fig. 1) [30].

\section{Animals}

Female BALB/c mice (5-6 weeks of age), female mongrel dogs (age $\sim 50$ weeks, weight $\sim 30 \mathrm{~kg}$ ), and female conventional pigs (age $\sim 13$ weeks, weight $\sim 40 \mathrm{~kg}$ ) were used with the approval of the IACUC of Seoul National University Hospital.

The BALB/c mice were anesthetized with $200 \mu 12.5 \%$ avertin solution (2,2,2-tribromoethanol-tert amyl alcohol; Sigma-Aldrich), and the imaging areas were treated with a depilatory cream.

For general anesthesia of dogs and pigs, $0.1 \mathrm{ml} / \mathrm{kg}$ zoletil (tiletamine $125 \mathrm{mg}$, zolazepam $125 \mathrm{mg} / \mathrm{ml}$; Virbac Korea) and $0.1 \mathrm{ml} / \mathrm{kg}$ Rompun (xylazine HCL $23.3 \mathrm{mg} / \mathrm{ml}$; Bayer Korea) were subcutaneously injected, followed by endotracheal intubation, ventilation with $100 \%$ oxygen, and maintenance with 1.5-2\% isoflurane. For the pigs, $0.25 \mathrm{mg} / \mathrm{kg}$ vecuronium was additively administered. For the animals that were kept alive for reexploration, cefazolin was administered subcutaneously before skin incision at a dose of $25-30 \mathrm{mg} / \mathrm{kg}$, and meloxicam was administered at a dose of $0.4 \mathrm{mg} / \mathrm{kg}$ subcutaneously before the recovery of anesthesia, as well as with a 1-mg tablet administered orally twice daily postoperatively.

\section{Methods}

\section{Optimal concentration for sentinel node mapping in the intestinal tract}

ICG $0.1 \mathrm{ml}$ was injected at the small bowel subserosally using a 25-gauge needle at different concentrations $(0.01,0.1,1$, and $5 \mathrm{mg} / \mathrm{ml}$ ), and the fluorescence signals were compared.

To simulate an endoscopic injection, the stomach was removed and opened along the greater curvature, and $0.1 \mathrm{ml} \mathrm{ICG}(0.001,0.01,0.1,1$, and $5 \mathrm{mg} / \mathrm{ml})$ was injected submucosally using a 25-gauge needle, followed by closure of the stomach. The magnitude of fluorescence signals at the injection sites and lymphatic flows were observed with $4 \mathrm{mmHg} / \mathrm{ml}$ pressure maintained inside the stomach using a laparoscopic $\mathrm{CO}_{2}$ inflator to simulate the pressure effects of gastroscopy (Supplementary Fig. 2).
Comparison of the retention time in the sentinel lymph nodes between tracers

Retention of tracers in the axillary lymph nodes in mice Three types of tracers ICG $(0.1 \mathrm{mg} / \mathrm{ml})$, ICG $/ \gamma-$ PGA complex (ICG $0.1 \mathrm{mg} / \mathrm{ml}, \gamma$-PGA $0.1 \%$ ), and NIR-PNG nanoprobe were injected intradermally into the forepaw pad of mice. The sentinel lymph node in the axillary area was observed by the NIR imaging system $1 \mathrm{~h}, 1,2$ days, and 1 week after the injection serially in the same mice, and the fluorescence intensity was compared. To observe histological findings, each tracer was injected in the same manner in three different groups of mice composed of four mice in each group, and the lymph node was harvested from the mice $1 \mathrm{~h}, 1,2$ days, and 1 week after the injection and observed by hematoxylin and eosin (H\&E) staining.

Retention of tracers in the small bowel and mesenteric lymph nodes in pigs To compare the retention time of tracers in the injected site and lymph nodes, three types of tracers were also injected into the small bowel of pigs, $0.1 \mathrm{ml}$ subserosally using a 25-gauge syringe, and the location of the injected site and lymph node was marked with metal clips and 4-0 prolene sutures. The signal intensity was evaluated 2 days or 1 week later by the NIR imaging system. Additionally, the dogs injected with each tracer into the stomach in later experiments were kept alive and reexplored 1 week later.

\section{Sentinel lymph node mapping in the stomach of large animals}

Canine model Three types of tracers ICG $(0.1 \mathrm{mg} / \mathrm{ml})$, ICG/ $\gamma$-PGA complex (ICG $0.1 \mathrm{mg} / \mathrm{ml}, \gamma$-PGA $0.1 \%$ ), and NIR-PNG nanoprobe were injected at a $0.2-\mathrm{ml}$ dose subserosally on the greater curvature side of the lower body of the stomach using a 25-gauge needle. The patterns of tracer movement and numbers of sentinel nodes and next lymph nodes were observed for more than $60 \mathrm{~min}$. The injection sites and sentinel nodes were marked with metal clips and reexplored 1 week later. In one dog, an endoscopic NIRPNG injection was followed by an injection of $0.5 \mathrm{ml}$ of $0.5 \mathrm{mg} / \mathrm{ml} \mathrm{ICG}$ in the same manner as in the experiments in pigs described earlier.

Porcine model A gastroscope (EG-450WR5 with an E400 system; Fujinon, Tokyo, Japan) was inserted via an overtube, and $0.2 \mathrm{ml}$ of three types of tracers was injected into the submucosal layer on the greater curvature side of the lower body of the stomach using a 23-gauge endoscopic injection needle (NM-400U-0423; Olympus, Aomori, Japan). After $30 \mathrm{~min}$ of observation of the tracer 

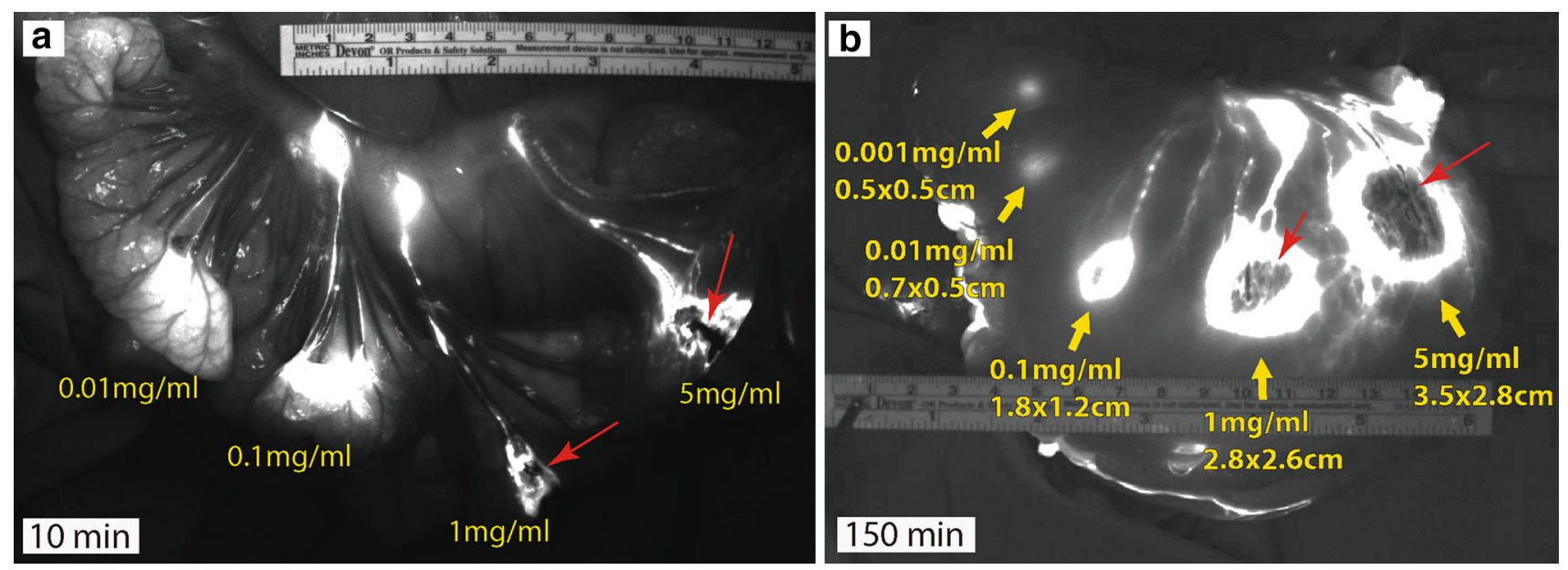

Fig. 1 Near-infrared (NIR) signals of indocyanine green (ICG) at different concentrations. a In vivo NIR images of the small bowel of a pig show bright signals in the lymph nodes with a $0.1 \mathrm{mg} / \mathrm{ml}$ or greater concentration of ICG. The signals were dark at first and then became brighter as ICG was diluted with 1 and $5 \mathrm{mg} / \mathrm{ml} \mathrm{ICG,} \mathrm{with} \mathrm{the}$ dark area remaining in the center of the injection site because of the

movement patterns and the numbers of lymph nodes, $0.5 \mathrm{ml}$ of $5 \mathrm{mg} / \mathrm{ml}$ ICG was injected at the same site in some pigs to compare the sensitivity and patterns of distribution both on NIR images and by visual inspection.

\section{Results}

Optimal concentration for sentinel lymph node biopsy in the intestinal tract

In vitro NIR signals of ICG in cryotubes were the brightest at a concentration of $0.01 \mathrm{mg} / \mathrm{ml}$ (Supplementary Fig. 2). When ICG was injected into the small bowel and the stomach, $0.01 \mathrm{mg} / \mathrm{ml}$ ICG seemed to be insufficient to reach the adjacent lymph nodes consistently. ICG at doses of 5 and $1 \mathrm{mg} / \mathrm{ml}$ did not show bright signals in vitro; however, ICG showed bright signals when injected in vivo, with a dark central area caused by a quenching effect (Fig. 1), and signals in the lymphatic vessels and lymph nodes in the small bowel were identified in a delayed manner: dark at first when the high concentration of ICG moves along the lymphatics and then becoming bright after dilution within minutes. The sizes of the injection sites at 5 and $1 \mathrm{mg} / \mathrm{ml}$ of ICG in the stomach were too large to specify the lymphatic flows from the injection sites compared with $0.1 \mathrm{mg} / \mathrm{ml} \mathrm{ICG} \mathrm{(Fig.} \mathrm{1b).} \mathrm{Therefore,} \mathrm{the} \mathrm{opti-}$ mal concentration, considered the lowest concentration to produce sufficient signal intensity with a small size of injection site signal, was chosen as $0.1 \mathrm{mg} / \mathrm{ml}$ for subsequent experiments. quenching effect (red arrows). b Ex vivo NIR image with sizes of injection sites in the stomach of a pig show that $0.1 \mathrm{mg} / \mathrm{ml}$ is the optimal concentration of ICG, with the smallest signal size of the injection site and sufficient signal for lymphatic flows with the least quenching effect

Signal intensity 1 week after injection into the small bowel of pigs

\section{Retention of tracers in axillary lymph node of mice}

As in our previous study, the ICG fluorescence signal was washed out $1 \mathrm{~h}$ after injection and had almost disappeared 1 day later. The ICG $\gamma$-PGA complex signal remained bright after $1 \mathrm{~h}$ and became faint 1 day later. However, the NIR-PNG signal was the brightest 1 day after injection and remained partially detectable in the lymph node until 1 week after injection in ex vivo images (Fig. 2). H\&E staining of the axillary lymph nodes, which were resected from other sets of mice $1 \mathrm{~h}, 1$ day, 2 days, and 1 week after injection of the tracers, showed no pathological abnormality regardless of the tracers used and time of resection (Supplementary Fig. 3).

\section{Retention of tracers in the small bowel mesenteric lymph nodes in pigs}

In contrast to the result in mice, the signal at the injection site and lymph nodes became very bright within a few minutes and remained bright until $1 \mathrm{~h}$ after injection regardless of the tracers used (Supplementary Fig. 4). Additionally, the signal of all the tracers remained in the injection site and lymph nodes in one pig 2 days after injection. Therefore, the subsequent experiments were designed to explore the abdomen of pigs 1 week after the injection. The signal intensity of ICG was faint or absent in the injection site and lymph nodes. The ICG/ $\gamma-\mathrm{PGA}$ 

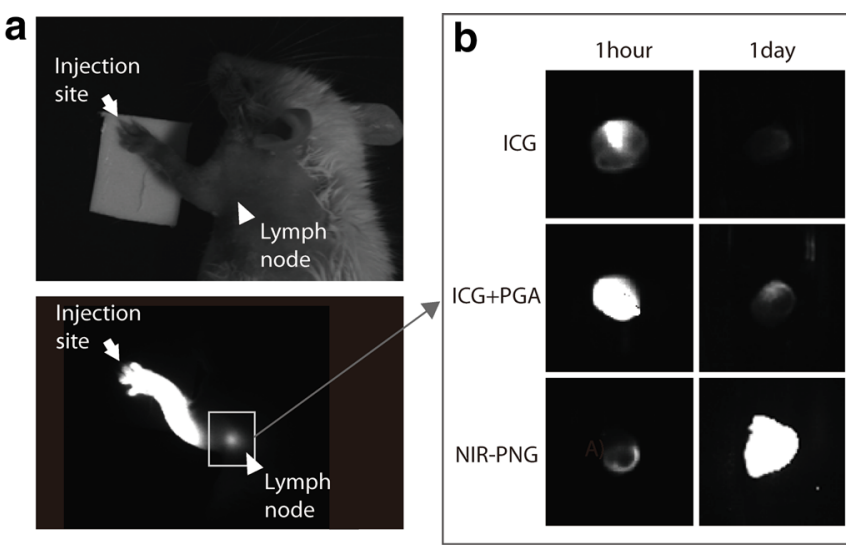

Fig. 2 a ICG, ICG/poly- $\gamma$-glutamic acid ( $\gamma$-PGA) complex, and nearinfrared polynagogel (NIR-PNG) was injected into the forepaw pad of mice and the axillary lymph node was observed by the NIR imaging system. b Ex vivo NIR images of the axillary sentinel lymph node

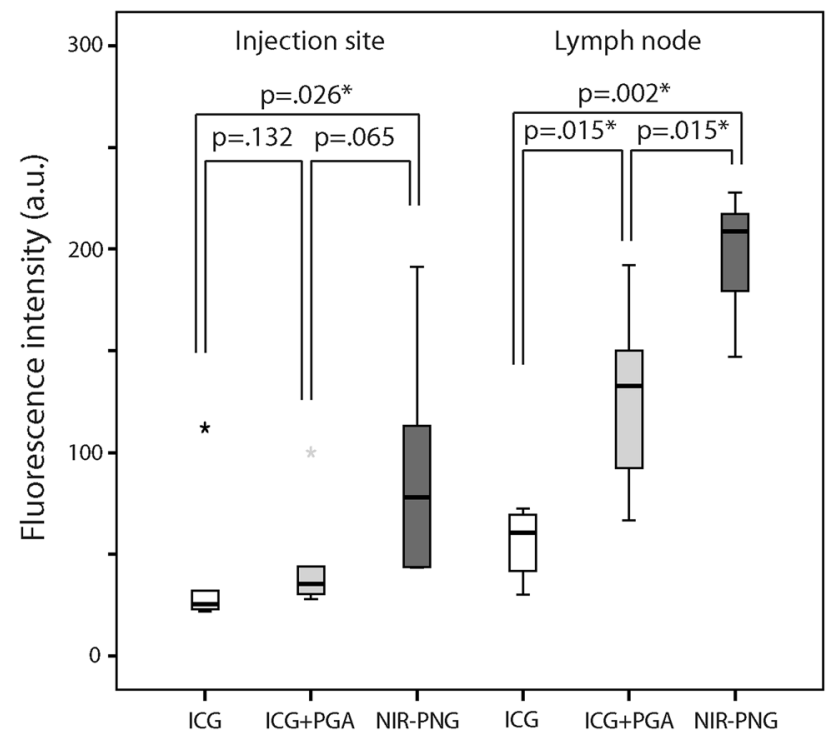

Fig. 3 Fluorescence intensities of injection sites and lymph nodes in the small bowel of pigs at 1 week after injection $(n=6)$. * $p$ value $\leq 0.05$ by the Mann-Whitney $U$ test

complex showed a faint signal in the injection site, but a bright signal remained partly in a small area of lymph nodes (Supplementary Fig. 5). NIR-PNG remained bright in the injection site in half the cases, and the signal in the lymph nodes was brighter and larger than that of ICG or ICG $/ \gamma$-PGA complex. The fluorescence measured in the lymph node also showed the highest intensity with NIRPNG, followed by ICG $/ \gamma$-PGA complex, and finally ICG with statistical significance (Fig. 3).

H\&E staining of the injection site showed mild to moderate inflammation in the subserosal area where the tracer was located without a significant difference between
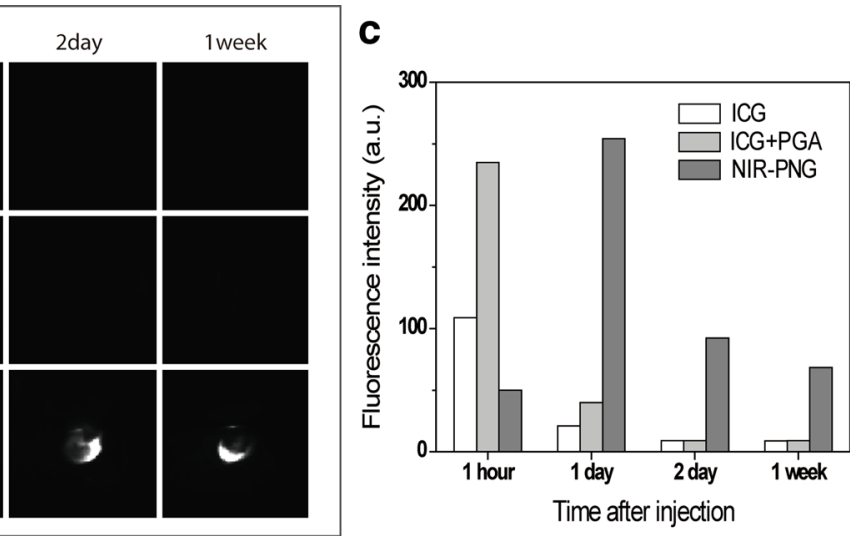

$1 \mathrm{~h}, 1$ day, 2 days, and 1 week after injection of each tracer. c Fluorescence intensities in lymph nodes according to tracer and observation time after injection

Table 1 Inflammation/fibrosis scores at injection site

\begin{tabular}{llllll}
\hline Pig no. & 1 & 2 & 3 & 4 & 5 \\
\hline ICG & $1 / 2$ & $1 / 2$ & $2 / 2$ & $1 / 2$ & $1 / 2$ \\
ICG + PGA & $1 / 2$ & $1 / 2$ & $2 / 2$ & $1 / 2$ & $1 / 2$ \\
NIR-PNG & $1 / 3$ & $1 / 2$ & $2 / 2$ & $1 / 2$ & $1 / 2$
\end{tabular}

ICG idocyanine, $P G A$ poly- $\gamma$-glutamic acid, NIR near-infrared, $P N G$ polymer nanogel 0 , no inflammation or fibrosis; 1 , mild; 2 , moderate; 3 , severe

tracers (Table 1). One sample injected with NIR-PNG showed severe fibrosis in the subserosal area, but all the other samples uniformly showed moderate fibrosis regardless of tracer. All the lymph nodes showed mild to moderate follicular hyperplasia without a significant difference between tracers (Supplementary Fig. 6).

Sentinel lymph node mapping in the stomach of dogs and pigs

\section{Canine stomach model}

Table 2 summarizes the fluorescence results in the stomach of dogs and pigs.

ICG and ICG/ $\gamma$-PGA complex quickly dispersed in multiple directions immediately after subserosal injection (Supplementary Fig. 7 A,B). The infrapyloric lymph node (sentinel node) was identified within several minutes, and the suprapancreatic lymph nodes beyond the sentinel node were also brightened. With NIR-PNG, either by subserosal or submucosal injection, no further lymph nodes were found beyond the sentinel node (Supplementary Fig. 7C, Table 2). The size of the NIR-PNG injection site was smaller than that 


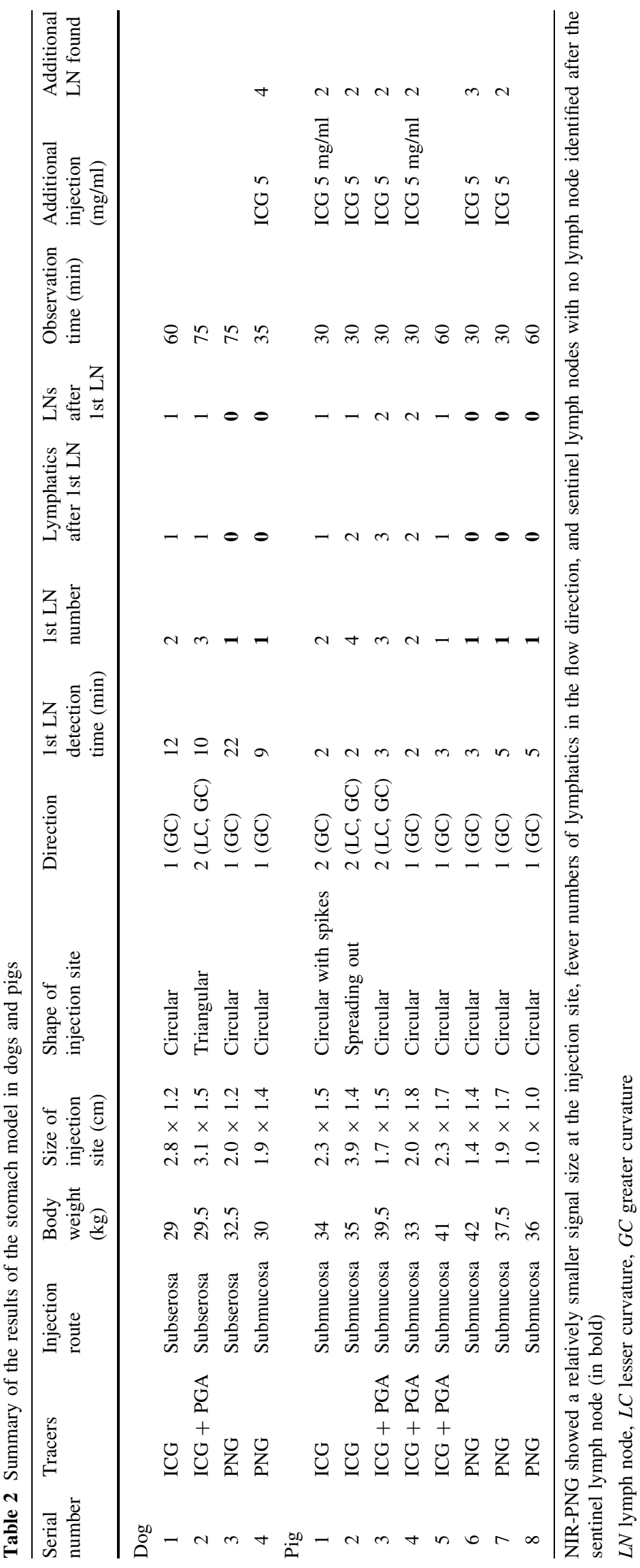


of ICG and ICG/ $\gamma$-PGA complex $(2.0 \times 1.2$ and $1.9 \times 1.4$ vs. $2.8 \times 1.2$ and $3.1 \times 1.5 \mathrm{~cm}$ ), which can be interpreted that NIR-PNG is more site specific. Injection of an additional $5 \mathrm{mg} / \mathrm{ml} \mathrm{ICG}$ in the final dog showed four additional lymph nodes beyond the sentinel node, confirming that the smaller number of sentinel lymph nodes with NIR-PNG resulted not from a paucity of lymph nodes in the pig but from the characteristic of NIR-PNG remaining in the sentinel nodes without further dispersion.

In the reexploration after 1 week, no fluorescence remained at either injection site or in the lymph nodes for ICG and ICG-PGA; however, the NIR-PNG signal was found at both sites.

\section{Porcine stomach model}

Table 2 summarizes the fluorescence results. Figure 4 and Supplementary Fig. 8 show images of porcine stomach injected with NIR-PNG and other tracers, respectively, followed by injection of ICG in $5 \mathrm{mg} / \mathrm{ml}$.

Similar to the results in the canine models, ICG and ICG $/ \gamma$-PGA complex spread to both the lesser and greater curvature sides of the stomach immediately after injection and showed multiple lymph nodes (Supplementary Fig. 8,B, Table 2). By contrast, NIR-PNG identified only one lymph node and did not move beyond it for more than
30 min (Fig. 4). When we compared NIR-PNG with other tracers in pigs, NIR-PNG showed a smaller number of total identified lymph nodes $(1.0 \pm 0.0$ vs. $3.8 \pm 1.3$, $p=0.021)$, the first lymph nodes $(1.0 \pm 0.0$ vs. $2.4 \pm 1.1$, $p=0.055)$, lymphatics after the first lymph nodes $(0 \pm 0$ vs. $1.4 \pm 0.55, p=0.018)$, and lymph nodes after the first lymph nodes ( $0 \pm 0$ vs. $1.8 \pm 0.84, p=0.020)$. The shape of the injection site was a spreading-out pattern with spikes and was larger with ICG and ICG/ $\gamma$-PGA, whereas it was circular and smaller in size with NIR-PNG.

Compared with the green color observed with the naked eye after injection of ICG at the same injection site with a concentration used for visual inspection $(5 \mathrm{mg} / \mathrm{ml})$, which causes difficulties in differentiating lymph nodes from venous vessels, NIR images allowed better tissue discrimination. NIR images of $5 \mathrm{mg} / \mathrm{ml}$ ICG revealed more lymph nodes beyond the first lymph node where NIR-PNG was indicated, indicting again that NIR-PNG has a greater tendency than ICG to remain in the sentinel lymph nodes and does not easily migrate to the lymphatics subsequently.

\section{Discussion}

NIR imaging technology has been proposed as a promising option that could replace vital dyes or radioisotopes for
Fig. 4 Sentinel lymph node $(S L N)$ mapping by endoscopic submucosal injection of NIRPNG in a gastric cancer model of pigs. Thirty minutes after injection of NIR-PNG, $0.5 \mathrm{ml}$ of $5 \mathrm{mg} / \mathrm{ml}$ ICG was injected at the same injection site, and the same area was observed by visual inspection and NIR imaging. Compared with ICG, which spreads widely and shows lymphatics and lymph nodes beyond the sentinel nodes, NIRPNG identified only one sentinel node. Yellow arrow sentinel lymph node, red arrowhead lymph node $(L N)$ beyond the sentinel lymph node
NIR-PNG $0.2 \mathrm{ml}$
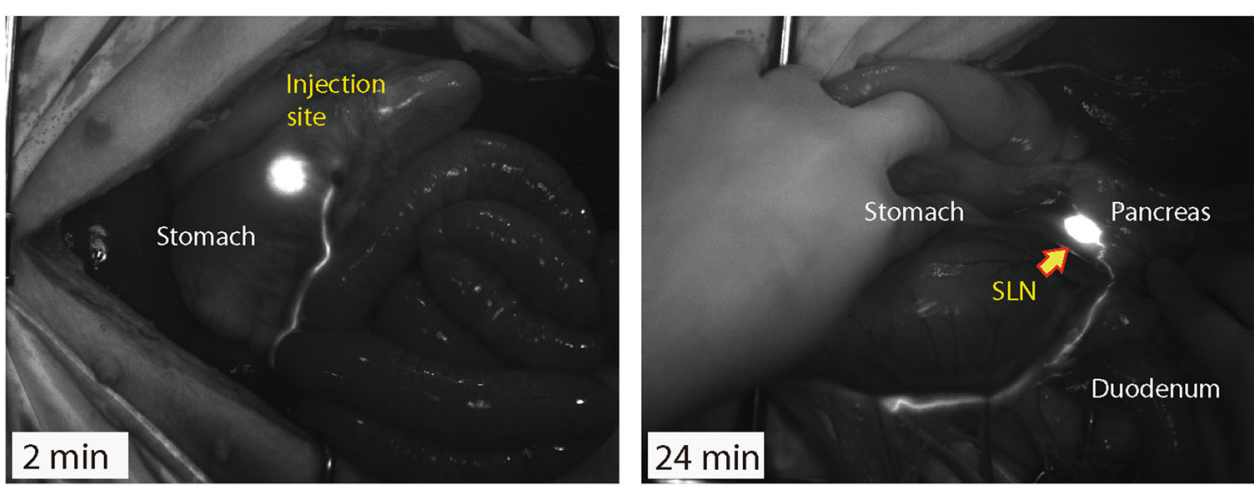

ICG $5 \mathrm{mg} / \mathrm{ml} 0.5 \mathrm{ml}$ at the same injection site
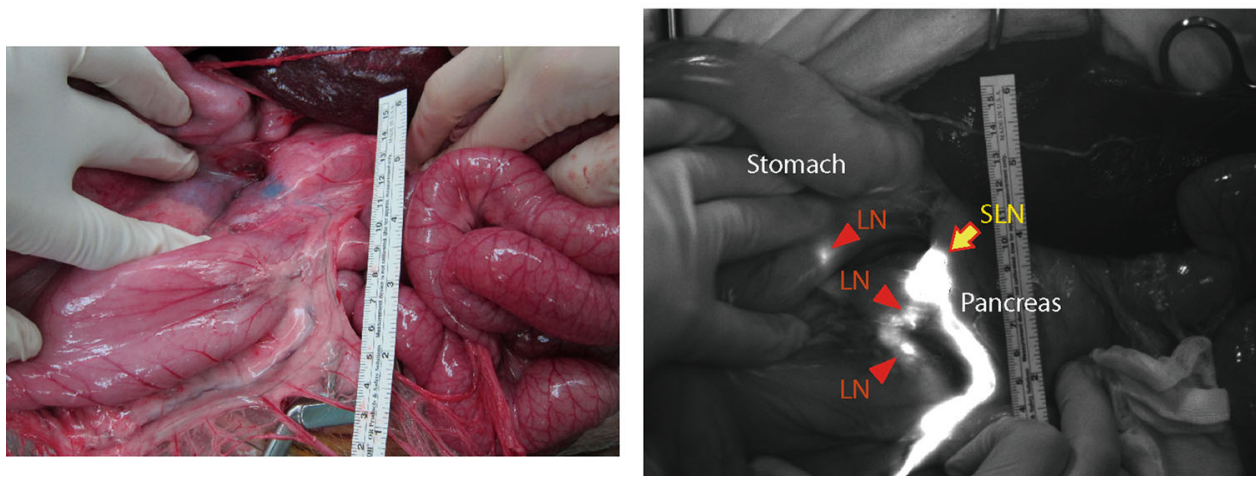
sentinel lymph node mapping because of its visibility and very good sensitivity without concern for radio-hazards. Inorganic fluorescent semiconductor nanocrystals called quantum dots have been reported to be good tracers with very high quantum yields and are likely to provide the capability to tune wavelengths in addition to their remarkable resistance to photobleaching. However, these tracers could not be readily applied in humans because of their possibly toxic cores [9-12]. By contrast, organic fluorophores, including ICG and modified heptamethine indocyanines, have lower quantum yields and higher susceptibility to photobleaching but better safety profiles. In particular, ICG was approved for indicator-dilution studies by the FDA in 1958, and it is known to be one of the least toxic dyes, with the only known adverse reaction being rare anaphylaxis [13].

The concentration of ICG commonly used in vital dye methods is too high for the NIR imaging system, because the rapid dispersion of ICG and high sensitivity of the NIR system showed too many lymph nodes [23, 31]. We have developed three strategies to overcome the weaknesses of ICG using materials that are possibly much less harmful than quantum dots.

The first method utilizes $0.1 \mathrm{mg} / \mathrm{ml}$ as the optimal concentration of ICG because it was found to be the smallest concentration to produce sufficient brightness in adjacent lymph nodes (Fig. 1). The amount injected also seemed to be sufficient at $0.2 \mathrm{ml}$, which is an amount approximately 100 times smaller than the conventional amount of ICG used in the vital dye method $(5 \mathrm{mg} / \mathrm{ml}$ and $0.5 \mathrm{ml})$ [21, 32, 33]. A smaller dose could be less toxic for a hypersensitivity reaction, a rare event for ICG, and also more specific because ICG at a greater dose spreads widely into multiple lymphatic flows [34]. However, $0.1 \mathrm{mg} / \mathrm{ml}$ ICG also spreads multi-directionally and beyond the sentinel nodes. Although the concentration of ICG could be further optimized in human applications considering the structural differences between human and animal stomach, it looks like very difficult to increase the specificity of sentinel node mapping by only tuning the concentration of ICG; in a previous human trial, ICG in $0.025 \mathrm{mg} / \mathrm{ml}$ was not sufficient and ICG in $0.05 \mathrm{mg} / \mathrm{ml}$ spreads quickly to multiple lymph nodes [23]. Nevertheless, diluted ICG seems to be clinically applicable because it clearly showed not only the lymph nodes but also the lymphatic channels, which could help surgeons to differentiate the sentinel lymph nodes from the lymph nodes beyond them.

The second strategy was to physically mix ICG with $\gamma$ PGA. Similar to our previous study that showed enhanced photostability and retention time in small animals [26], the ICG $\gamma$-PGA complex showed a brighter signal in the axillary lymph node in mice than ICG, and remained longer in the small bowel mesenteric lymph node in pig. A long retention time in the lymph node indirectly means that the tracer is not quickly drained through the lymphatic vessels distal to the lymph node. However, in the stomach of large animals, the pattern of ICG/ $\gamma$-PGA complex distribution was not much different from that of ICG. The most probable explanation is that, because the complex is derived by physical mixing, free ICG from the complex can travel quickly to distal lymphatic channels, and the very sensitive NIR imaging system detects the signal of free ICG. Conversely, it is also possible that ICG functions similarly to the ICG $/ \gamma$-PGA complex as the result of abundant lipoproteins or albumin in the lymphatic system around the gastrointestinal tract and could play a role similar to $\gamma$-PGA to bind ICG with high affinity [35]. This hypothesis also explains the sustained signals in the small bowel found 2 days after injections with ICG, the signal intensity of which usually diminishes rapidly and is depleted within $24 \mathrm{~h}$ in vitro [20, 33]. Nevertheless, a significantly brighter and longer signal observed in the small bowel of pigs 1 week after injection implies a potential benefit of the ICG/ $\gamma$-PGA complex compared to ICG alone in human application because the ICG $/ \gamma$-PGA complex can provide more options regarding when to inject the tracers before operation.

The third strategy was to manufacture the nanoparticle with pullulan and cholesterol conjugated with IRDye800. This nanogel is an ideal size $(\sim 30 \mathrm{~nm})$ for sentinel node mapping, [36] and moved to the first lymph node and did not move beyond in all five animals tested, whereas every other animal injected with ICG or ICG/ $\gamma$-PGA complex showed at least one additional lymph node beyond the first lymph nodes. Additionally, the signal at the injection site did not have an out-spreading shape and was smaller than those with ICG and ICG/ $\gamma$-PGA complex. A relatively smaller injection site size can be helpful to identify sentinel nodes that are more specific to the primary lesion.

Combining the results of the long retention time in the small bowel lymph node and the experiments in the stomach, NIR-PNG showed ideal characteristics as a tracer for use in sentinel node navigation surgery because it stays only in the sentinel lymph node and does not move to consecutive distal lymph nodes, which characteristics can minimize "false" sentinel lymph nodes and additional effort and time to evaluate the nodes. The characteristic of the longer retention time of NIR-PNG in the sentinel node, with no flow beyond, seems to support a wide range of injection times before surgery, with more consistent results of sentinel node mapping than with ICG.

Regarding sensitivity, a smaller number of sentinel nodes could be disadvantageous [37]. Therefore, we injected $5 \mathrm{mg} / \mathrm{ml}$ ICG at the same site as NIR-PNG in pigs to examine whether only one lymph node detected by NIRPNG resulted from low sensitivity. Additional injection 
revealed additional lymph nodes beyond the lymph node identified by NIR-PNG, but no additional nodes were identified between the injection site and sentinel node. This result supports the concept that detection of only one sentinel node resulted not from an inferior sensitivity of NIR-PNG but from the beneficial characteristic of NIRPNG to remain in the first lymph node and a smaller number of perigastric lymph nodes in animal models compared with the human stomach. Because actual sensitivity and specificity of sentinel node tracers only can be accurately assessed in lymph node-positive cancer patients, further comparative studies with radioisotopes in humans may answer the question if NIR imaging can completely replace radioisotope methods.

The safety of retained tracers is an issue that must be addressed, particularly because this problem could occasionally arise in clinical settings. Pullulan, the backbone material in NIR-PNG, was approved as a food ingredient in Japan in 1976 and as a substance generally recognized as safe (GRAS) by the FDA in 2002. Because of the nontoxic and nonimmunogenic properties of pullulan, NIR-PNG could have a better safety profile than quantum dots [24, 25]. Although our study used a limited number of samples, histological examination of the injection sites and lymph nodes in mice and the small bowel of pigs did not show a significant difference between tracers, except for the increased fibrosis in one sample injected with NIR-PNG. However, the relative increase in fibrosis might not pose a clinically significant risk compared with the moderate fibrosis in the other samples. The follicular hyperplasia found in the lymph nodes at 1 week after injection could be related to a foreign-body reaction or to the injection procedure itself. Although the long-term safety of retained tracers in the body should be further investigated, our study at least showed that the findings for the ICG $/ \gamma-\mathrm{PGA}$ complex and the NIR-PNG nanogel were similar to those for ICG using a very small concentration.

In summary, NIR-PNG could be a good candidate for sentinel node mapping in gastric cancer because it demonstrates good sensitivity, specificity, and a long retention time in the sentinel nodes. Diluted ICG and its physical mixture with the $\gamma$-PGA complex, which resulted in a longer retention time, could also be utilized as inorganic fluorophore tracers that are less toxic than quantum dots, because they provide highly sensitive visualization of not only the lymph nodes but also lymphatic flows, findings that can help surgeons identify the sentinel nodes.

Acknowledgments This study was supported by SNUH research funding (nos. 30-2010-0070 and 04-2012-0690). We appreciate all the invaluable support and assistance to the animal experiments from our colleagues including Dong-Seok Han, Kyung-Goo Lee, Seung-Young Oh, Jun-Young Yang, Sebastian Kwon, Tae-Su Han, Jieun Yoo and Boram Choi in Department of Surgery and Laboratory of Gastric
Cancer Biology, Young-Hwa Kim in Department of Nuclear Medicine, Sun-Kwon Kim in Department of Biomedical Engineering, and Sang-Young Park and Sa-Hun Kim, D.V.M.s in the Department of Experimental Animal Research.

\section{References}

1. Moon HG, Han W, Noh DY. Comparable survival between pN0 breast cancer patients undergoing sentinel node biopsy and extensive axillary dissection: a report from the Korean Breast Cancer Society. J Clin Oncol. 2010;28:1692-9.

2. Wong SL, Balch CM, Hurley P, et al. Sentinel lymph node biopsy for melanoma: American Society of Clinical Oncology and Society of Surgical Oncology joint clinical practice guideline. J Clin Oncol 2012;30:2912-2918

3. Miyashiro I. What is the problem in clinical application of sentinel node concept to gastric cancer surgery? J Gastric Cancer. 2012;12:7-12.

4. Miwa K, Kinami S, Taniguchi K, Fushida S, Fujimura T, Nonomura A. Mapping sentinel nodes in patients with early-stage gastric carcinoma. Br J Surg. 2003;90:178-82.

5. Park DJ, Lee HJ, Lee HS, et al. Sentinel node biopsy for cT1 and cT2a gastric cancer. Eur J Surg Oncol. 2006;32:48-54.

6. Hayashi H, Ochiai T, Mori M, et al. Sentinel lymph node mapping for gastric cancer using a dual procedure with dye- and gamma probe-guided techniques. J Am Coll Surg. 2003; 196:68-74.

7. Kitagawa Y, Fujii H, Mukai M, Kubota T, Otani Y, Kitajima M. Radio-guided sentinel node detection for gastric cancer. $\mathrm{Br} \mathrm{J}$ Surg. 2002;89:604-8.

8. Tonouchi H, Mohri Y, Tanaka K, Kobayashi M, Ohmori Y, Kusunoki M. Laparoscopic lymphatic mapping and sentinel node biopsies for early-stage gastric cancer: the cause of false negativity. World J Surg. 2005;29:418-21.

9. Iga AM, Robertson JH, Winslet MC, Seifalian AM. Clinical potential of quantum dots. J Biomed Biotechnol. 2007;2007:76087.

10. Kim S, Lim YT, Soltesz EG, et al. Near-infrared fluorescent type II quantum dots for sentinel lymph node mapping. Nat Biotechnol. 2004;22:93-7.

11. Buckle T, Chin PT, van Leeuwen FW. (Non-targeted) radioactive/fluorescent nanoparticles and their potential in combined preand intraoperative imaging during sentinel lymph node resection. Nanotechnology. 2010;21:482001.

12. Pons T, Pic E, Lequeux N, et al. Cadmium-free $\mathrm{CuInS}_{2} / \mathrm{ZnS}$ quantum dots for sentinel lymph node imaging with reduced toxicity. ACS Nano. 2010;4:2531-8.

13. Benson RC, Kues HA. Fluorescence properties of indocyanine green as related to angiography. Phys Med Biol. 1978;23:159-63.

14. Kitai T, Inomoto T, Miwa M, Shikayama T. Fluorescence navigation with indocyanine green for detecting sentinel lymph nodes in breast cancer. Breast Cancer. 2005;12:211-5.

15. Troyan SL, Kianzad V, Gibbs-Strauss SL, et al. The FLARE intraoperative near-infrared fluorescence imaging system: a firstin-human clinical trial in breast cancer sentinel lymph node mapping. Ann Surg Oncol. 2009;16:2943-52.

16. Tagaya N, Aoyagi H, Nakagawa A, et al. A novel approach for sentinel lymph node identification using fluorescence imaging and image overlay navigation surgery in patients with breast cancer. World J Surg. 2011;35:154-8.

17. Noura S, Ohue M, Seki Y, et al. Feasibility of a lateral region sentinel node biopsy of lower rectal cancer guided by indocyanine green using a near-infrared camera system. Ann Surg Oncol. 2010;17:144-51. 
18. Hirche C, Mohr Z, Kneif S, et al. Ultrastaging of colon cancer by sentinel node biopsy using fluorescence navigation with indocyanine green. Int J Colorectal Dis. 2012;27:319-24.

19. Crane LM, Themelis G, Pleijhuis RG, et al. Intraoperative multispectral fluorescence imaging for the detection of the sentinel lymph node in cervical cancer: a novel concept. Mol Imaging Biol (MIB). 2011;13:1043-9.

20. Miyashiro I, Miyoshi N, Hiratsuka M, et al. Detection of sentinel node in gastric cancer surgery by indocyanine green fluorescence imaging: comparison with infrared imaging. Ann Surg Oncol. 2008;15:1640-3.

21. Kusano M, Tajima Y, Yamazaki K, Kato M, Watanabe M, Miwa M. Sentinel node mapping guided by indocyanine green fluorescence imaging: a new method for sentinel node navigation surgery in gastrointestinal cancer. Dig Surg. 2008;25:103-8.

22. Tajima Y, Yamazaki K, Masuda Y, et al. Sentinel node mapping guided by indocyanine green fluorescence imaging in gastric cancer. Ann Surg. 2009;249:58-62.

23. Yoshida M, Kubota K, Kuroda J, et al. Indocyanine green injection for detecting sentinel nodes using color fluorescence camera in the laparoscopy-assisted gastrectomy. J Gastroenterol Hepatol. 2012;27(Suppl 3):29-33.

24. Akao T, Kimura T, Hirofuji YS, et al. A poly(gamma-glutamic acid)-amphiphile complex as a novel nanovehicle for drug delivery system. J Drug Target. 2010;18:550-6.

25. Lee EH, Kamigaito Y, Tsujimoto T, Uyama H, Sung MH. Synthesis of an amphiphilic poly(gamma-glutamic acid)-cholesterol conjugate and its application as an artificial chaperone. J Microbiol Biotechnol. 2010;20:1424-9.

26. Noh YW, Park HS, Sung MH, Lim YT. Enhancement of the photostability and retention time of indocyanine green in sentinel lymph node mapping by anionic polyelectrolytes. Biomaterials. 2011;32:6551-7.

27. Saxena V, Sadoqi M, Shao J. Polymeric nanoparticulate delivery system for indocyanine green: biodistribution in healthy mice. Int J Pharm. 2006;308:200-4.
28. Proulx ST, Luciani P, Derzsi S, et al. Quantitative imaging of lymphatic function with liposomal indocyanine green. Cancer Res. 2010;70:7053-62.

29. Cheng KC, Demirci A, Catchmark JM. Pullulan: biosynthesis, production, and applications. Appl Microbiol Biotechnol. 2011;92:29-44.

30. Noh YW, Kong SH, Choi DY et al. Near-infrared emitting polymer nanogels for efficient sentinel lymph node mapping. ACS Nano 2012;6:7820-7831

31. Miyashiro I, Kishi K, Yano M, et al. Laparoscopic detection of sentinel node in gastric cancer surgery by indocyanine green fluorescence imaging. Surg Endosc. 2011;25:1672-6.

32. Park do J, Kim HH, Park YS, et al. Simultaneous indocyanine green and $(99 \mathrm{~m}) \mathrm{Tc}$-antimony sulfur colloid-guided laparoscopic sentinel basin dissection for gastric cancer. Ann Surg Oncol. 2011;18:160-5.

33. Tajima Y, Murakami M, Yamazaki K, et al. Sentinel node mapping guided by indocyanine green fluorescence imaging during laparoscopic surgery in gastric cancer. Ann Surg Oncol. 2010;17:1787-93.

34. Cimmino VM, Brown AC, Szocik JF, et al. Allergic reactions to isosulfan blue during sentinel node biopsy-a common event. Surgery (St. Louis). 2001;130:439-42.

35. Yoneya S, Saito T, Komatsu Y, Koyama I, Takahashi K, DuvollYoung J. Binding properties of indocyanine green in human blood. Invest Ophthalmol Vis Sci. 1998;39:1286-90.

36. Henze E, Schelbert HR, Collins JD, Najafi A, Barrio JR, Bennett LR. Lymphoscintigraphy with Tc-99m-labeled dextran. J Nucl Med. 1982;23:923-9.

37. Ryu KW, Eom BW, Nam BH, et al. Is the sentinel node biopsy clinically applicable for limited lymphadenectomy and modified gastric resection in gastric cancer? A meta-analysis of feasibility studies. J Surg Oncol. 2011;104:578-84. 